Maziarz Barbara, Chojęta Dariusz, Zygmunt Ewelina, Wróblewski Hubert, Zimna Aleksandra. Influence of vegan diet on physical performance of athletes. Journal of Education, Health and Sport. 2020;10(7):209-215. eISSN 2391-8306. DOI http://dx.doi.org/10.12775/JEHS.2020.10.07.023

https://apcz.umk.pl/czasopisma/index.php/JEHS/article/view/JEHS.2020.10.07.023

https://zenodo.org/record/3960101

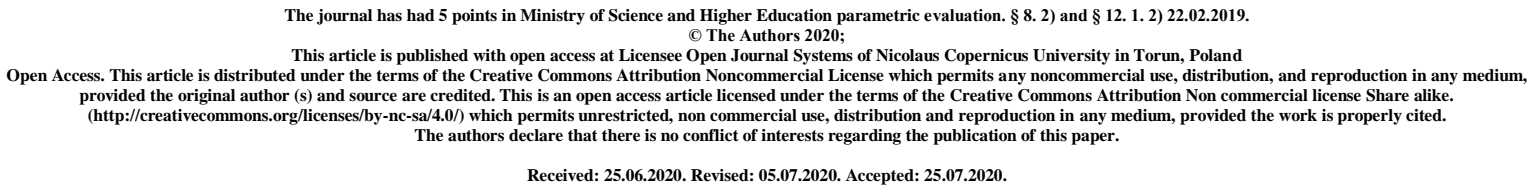

\title{
Influence of vegan diet on physical performance of athletes
}

\author{
Barbara Maziarz $^{1}$, Dariusz Chojęta ${ }^{1}$, Ewelina Zygmunt ${ }^{1}$, Hubert Wróblewski ${ }^{1}$, \\ Aleksandra Zimna ${ }^{1}$ \\ ${ }^{1}$ Faculty of Medicine, Medical University of Lublin, Chodźki Street 19, 20-093 Lublin, Poland
}

\begin{abstract}
Vegetarian and vegan diets are becoming more and more popular. Vegetarianism is a diet that eliminates all kinds of meat from the diet, including poultry, fish and seafood. Vegans give up on eating not only meat, but also other products of animal origin, the production of which is associated with the exploitation of animals, such as: dairy products (including eggs) and honey. It is not only a diet, but above all a lifestyle that includes many aspects such as not using cosmetics or buying clothes made of animal raw materials, not participating in entertainment in which animals are used. A vegan diet should be properly balanced, because people using it are more likely to be deficient in vitamin B12, iron, and calcium. The effect of a vegan diet on the growth of muscle mass in athletes, including bodybuilders, has also been observed. The aim of the study was to determine the effect of a vegan diet on the results achieved in body sports and to compare this information with non-vegan diets. The work is illustrative. The article is based on a review of the current literature available in the PubMed database, information and research from the Internet and documentary programs.
\end{abstract}

Key words: vegan diet; sport; bodybuilding; nutrition 


\section{Introduction:}

A vegan diet is about the complete elimination of animal products. It carries the risk of deficiencies of various important vitamins and macronutrients [1]. Vitamin B12, iron and calcium are particularly important here $[1,2]$. The reduced concentration of these substances reduces the performance of athletes and may cause anemia and other diseases of the haematopoietic system. Also, low calcium concentration is associated with an increased risk of fractures and lower mechanical strength of the bones. That is why supplementation and properly balanced meals are so important. The impact of a vegan diet on the cardiovascular system and blood lipid levels is also important [3-7]. The article will also discuss the relationship of this type of nutrition with muscle mass gain and the influence of oxidative stress on myocytes, an aspect particularly important for bodybuilders and typical strength athletes.

\section{Advantages and disadvantages of a vegan diet}

There are many myths about the vegan diet. It is popular to say that a vegan diet is deficient. It is worth mentioning here about the UK study, published in 2002, which is the largest study comparing the diet and lifestyle of vegetarians and carnivores. The study included a total of almost 34 thousand. people eating everything (including meat), over 21 thousand. vegetarians and 10,000 pescowegetarian. This study found that vegans consume more fiber, vitamin. B1, $\mathrm{C}, \mathrm{E}$, magnesium, folate and - iron. Based on the above work, it turned out that this diet is deficient in the three components of calcium, as well as vitamins B12 and D. However, the results of omnivores proved to be more disturbing. There were as many as 7 deficient components: calcium, dietary fiber, vitamins C, E, D, as well as magnesium and folates.

Research by Haddad et al. [8] assessed the nutritional status of adult vegans in comparison with omnivores. Vegans have been shown to have a protein content significantly lower than those of meat eaters. Vegans had lower blood levels of saturated and monounsaturated fats, as well as deficiencies in iron, calcium, zinc, vitamin D, vitamin B12, and amino acids; on the other hand, they showed higher levels of fiber and most nutrients such as ascorbic acid, folic acid and (copper, magnesium and manganese). In addition, vegans also showed lower levels of leukocytes, lymphocytes, platelets, complement factor 3, and blood urea nitrogen, but higher levels of albumin.

In 2018, a paper on the impact of a vegan diet on muscle mass, oxidative status and myocyte differentiation was published [9]. The effects of serum on the oxidative, growth and differentiation of both the $\mathrm{H} 9 \mathrm{c} 2$ and $\mathrm{H}-\mathrm{H} 9 \mathrm{c} 2$ cardiomyoblast cell lines were compared, and the study showed that treatment with vegan sera of both $\mathrm{H} 9 \mathrm{c} 2$ and $\mathrm{H}-\mathrm{H} 9 \mathrm{c} 2$ cells induced an increase in TBARS, a marker of lipid peroxidation, compared to vegetarian sera and omnivores. Increased lipid oxidation can be caused by the high doses of antioxidant molecules, vitamin $\mathrm{C}$, vitamin $\mathrm{E}$, and carotenoids present in a vegan diet. We observed a significant decrease in free nitric oxide in the medium treated with vegan and vegetarian $\mathrm{H}$ H9c2 cells with a concomitant increase in cell death compared to omnivorous serum. Under cellular oxidative stress, the amount of diffusive free NO decreased because it was converted to peroxynitrite (ONOO -) when it reacted with superoxide radicals. The study showed that long-term consumption of a strict vegan diet can neither protect nor prevent oxidative damage, nor inhibit chronic disease and maintain a healthy biological system. 


\section{Basics of a balanced vegan diet}

$\underline{\text { Cobalamin }}$

Cobalamin, or vitamin B12, is a group of water-soluble chemical compounds containing cobalt as the central atom. It is a coenzyme of many important reactions in the human body, ranging from the synthesis and regulation of DNA and red blood cells, to the metabolism of amino acids and lipids. The active forms of vitamin B12 are adenosylcobalamin and methylcobalamin, which are its coenzymatic forms. Cyanocobalamin is a very stable form of the vitamin, and it is easily converted to other forms in the human body. Both plant and animal organisms are unable to synthesize B12. Bacteria and archaea produce them. The accumulated "stocks" are sufficient to meet the needs of adults for 2-5 years. The daily requirement for this vitamin according to the Institute of Food and Nutrition is on average 2.4 $\mu \mathrm{g}$. Vitamin B12 is the only chemical compound that cannot be found in commonly consumed and available plants.

Symptoms of deficiency are primarily megaloblastic anemia and its symptoms (weakness, fatigue, dizziness, pallor). On the part of the nervous system, symptoms may also include weakness, postural disturbances and uncertainty in movement. There are also mental disorders, even dementia and depression.

Hence, its supplementation is so important for athletes. Deficiencies result in reduced endurance and exercise tolerance.

Research has begun on the use of plant foods to increase Cbl consumption [10, 11]. Some seaweed, mushrooms, and fermented foods can be a useful source of $\mathrm{Cbl}$, but data is still scarce and production is too heterogeneous.

So, at the moment the most effective method is supplementation of vitamin B12 in the form of tablets. According to Carmel, a single oral dose of $50 \mu \mathrm{g}, 500 \mu \mathrm{g}$ or $1000 \mu \mathrm{g}$ will be absorbed in an amount of $1.5 \mu \mathrm{g}, 9.7 \mu \mathrm{g}$ or $13 \mu \mathrm{g}$, respectively. To meet the daily $\mathrm{Cbl}$ requirement, one oral dose of 50-100 mcg / day or $2000 \mathrm{mcg}$ per week divided into two oral doses of cyanocobalamin may be sufficient to meet the needs of $2.4 \mathrm{mcg} /$ day for healthy adult vegetarians in terms of absorption efficiency.

$\underline{\text { Vitamin D }}$

Vitamin D is a fat-soluble vitamin, and its two forms - D2 - ergocalciferol and D3 cholecalciferol - are stored mainly in adipose tissue and skeletal muscles of our body.

Vitamin D is responsible for the calcium-phosphate metabolism, disturbances of which may lead to bone demineralization, and further to osteomalacia and osteoporosis. Its deficiency may also increase the risk of cardiovascular diseases, diabetes, and even autoimmune diseases and cancer.

Hence, its supplementation in athletes is so important [12,13]. Deficiencies can reduce bone strength and low-energy fractures, especially in competitive sports.

Most plant-based foods do not contain vitamin D at all. The exception is mushrooms, and more specifically champignons, in which there are 1.94 micrograms of this vitamin per 100 grams.

However, the basic source of vitamin D is its skin synthesis, which occurs under the influence of UVB rays with a wavelength of $280-315 \mathrm{~nm}$. Cholecalciferol is synthesized from 7- dehydrocholesterol, a derivative of the known cholesterol. However, in the fall and winter months, its supplementation is recommended, especially in vegans. 


\section{Protein}

For athletes, a properly balanced diet is extremely important. Data indicate that protein requirements should be aligned with athletic requirements. Typical recommendations include $1.6-1.7 \mathrm{~g} / \mathrm{kg} /$ day for strength athletes and $1.2-1.4 \mathrm{~g} / \mathrm{kg} /$ day for competitive athletes [14]. For most inactive people, $0.8 \mathrm{~g} / \mathrm{kg} /$ day is recommended. Protein consumption by vegans requires attention to the quantity and quality of protein consumed. It is often incomplete and does not contain all the essential amino acids. So the variety of foods you eat is very important. The Academy of Nutrition and Dietetics (AND) has recommended that vegans consume a variety of plant-based proteins to meet their protein and amino acid requirements. Foods such as grains, legumes, nuts and seeds should be included in the diet to ensure that all essential amino acids (EAAs) are present and that you consume adequate amounts of BCAAs to aid recovery and adaptation after training.

\begin{tabular}{|c|c|}
\hline Products & The amount of protein in 100g \\
\hline Pumpkin seeds & 30.2 \\
\hline Lentils (red, uncooked) & 24.6 \\
\hline Black beans (uncooked) & 21.6 \\
\hline Almonds & 21.2 \\
\hline Tempeh & 20.3 \\
\hline Tofu & 17.3 \\
\hline Oatmeal & 16.9 \\
\hline Quinoa (uncooked) & 14.1 \\
\hline
\end{tabular}

Table 1. Protein content in 100g of various vegan products (Based on Glick-Bauer M., Yeh M., 2014)

Athletes, especially those specializing in strength exercises, and bodybuilders often use protein isolates. Wholesome soy protein is popular, but also mixtures of pea protein isolates or brown rice.

\section{Carbohydrates}

They are the main source of energy, therefore they are very important for athletes. Research suggests that some endurance athletes may deliberately adopt a vegan diet to meet their carbohydrate needs or help them gain a certain weight. 8 In an adult diet, carbohydrates should be between 50 and 70\%, including sugars 10-20\% and added sugars below $10 \%$ of the total daily energy requirement. The sources of these organic compounds in a vegetarian diet are: dry legume seeds, unprocessed grain products, vegetables and fruits. Hence the fact that a vegetarian diet is by definition a diet high in carbohydrates. In fact, switching to a vegan diet eliminates only lactose from carbohydrates, which is neither essential nor nutritionally important sugar. It is also high in fiber and SFA, and does not contain much cholesterol. It is a diet with high nutritional density and therefore promotes weight loss. In addition, this diet regulates the lipid metabolism, and therefore reduces the risk of cardiovascular diseases. 
$\underline{\text { Fats }}$

Vegan diets are lower in total fat and saturated fat [9], and are also richer in $\Omega-6$ fats. Studies have shown that a low-fat diet can negatively affect testosterone levels in men. Reaching recommended values of $0.5-1.5 \mathrm{~g} / \mathrm{kg} /$ day (or $30 \%$ of the daily caloric intake) is possible for vegan athletes who consume the right amount of oil, avocado, nuts or seeds.

\section{The influence of a vegan diet on sports achievements}

The so-called vegan sport is becoming more and more popular. Many athletes declare that they are vegan and attribute their success to switching to a vegan diet. Patrik Baboumian, the strongest vegan in the world, proves that you can be vegan and strongman at the same time. Patrik Baboumian is the winner of the 2011 German strongman competition.

Being already a vegan, in 2012 he won the European title in powerlifting and broke the Guinness record in strength disciplines. He admits that he achieved his best sports results thanks to a vegan diet. In one of the interviews he said: "When you are an athlete, proving that a vegetarian or, in my case, a vegan diet is healthy is easier. This can be shown on the basis of sports achievements. If the sports results you get while on a vegan diet are better than those when you didn't follow a vegan or vegetarian diet, I think this is the best answer to the question of whether such a diet is healthy, he admitted. When I became vegetarian, my athletic performance improved by about $25 \%$. Once I became vegan my results improved again".

Scott Gordon Jurek (born October 26, 1973) - American ultramarathon runner, since 1997 he has been a vegetarian, and since 1999 a vegan. He has numerous achievements and medals to his credit.

Fiona Oakes is a British long-distance runner who holds four marathon world records. Oakes has been a vegan since she was 6 years old.

David Carter - American NFL defender of American football and vegan activist.

These are just a few of the many world-renowned athletes who are vegan. As can be seen, this diet has a positive effect on the results in strength and endurance sports $[15,16]$. There have been many studies assessing the effect of a vegan diet on the body's performance.

Endurance athletes have a different need than strength athletes.Some studies assess the potential of diet to indirectly impact performance by measuring maximum oxygen uptake, strength, blood acid-base status, acute MPS, and chronic muscle growth rather than actual performance at sporting events. while others measure performance results, such as time to exhaust when cycling. A review by Craddock and colleagues [17] summarized most of the literature to date on vegetarian diets and strength, anaerobic and aerobic exercise. It included 8 studies. Three of these studies focused on strength training and strength training, four assessed both anaerobic and aerobic performance, and only one investigated the effects of endurance exercise on immune markers. There was no difference in strength, anaerobic or aerobic. It was found that exercise performance did not appear to differ between dietary 
groups in terms of multiple measures and types of activity. Only the lower total creatine content in the body and the plasma carnitine in vegetarians seem to be important. Vegans are most likely to experience greater gains in performance when loaded with creatine in adenosine triphosphate / creatine phosphate-based activities.

On the other hand, a study comparing vegetarian and omnivorous adult endurance athletes showed higher maximum values of oxygen uptake by vegetarians and comparable strength [18].

\section{Summary}

The vegan diet is becoming more and more popular among athletes, including professional ones. As the research and literature review described above show, it has a beneficial effect on many aspects of health. Also, many world-famous athletes admit its beneficial influence on the achieved results. However, as research shows, there are no significant differences in strength, anaerobic or aerobic strength, or endurance. A greater increase in performance after creatine loading was observed in activities based on the adenosine triphosphate / phosphocreatine system. This is explained by the lower starting concentration of creatine in vegans. Although much work has been done and much research has been done comparing vegan athletes to people who eat animal products, the differences in performance and strength remain an area of interest.

\section{Bibliography:}

1. Rogerson D. Vegan diets: practical advice for athletes and exercisers. J Int Soc Sports Nutr 2017 Sep 13

2. Appleby PN, Key TJ. The long-term health of vegetarians and vegans. Proc Nutr Soc. 2016;75:287-293

3. Appleby, P. N., Key, T. J., Thorogood, M., Burr, M. L. and Mann, J. (2002). Mortality in British vegetarians. Public Health Nutr. 5:29-36.

4. Fraser G, Katuli S, Anousheh R, et al. (2015) Vegetarian diets and cardiovascular risk factors in black members of the Adventist Health Study-2. Public Health Nutr 18, 537545

5. Larpin C,Wozniak H, Genton L, Serratrice J. Vegetarian and vegan diets and their impact on health. Rev Med 2019 Oct 16; 15(667):1849-1853

6. Dinu M,Abbate R,Gensini GF,Casini A, Sofi F. Vegetarian, vegan diets and multiple health outcomes: A systematic review with meta-analysis of observational studies.Critical Reviews in Food Science and Nutrition. 2016 57: 3640-3649

7. Key TJ, Appleby PN, Rosell MS.Health effects of vegetarian and vegan diets.Proc Nutr Soc. 2006 Feb;65(1):35-41.

8. Haddad, E. H., Berk, L. S., Kettering, J. D., Hubbard, R. W., \& Peters, W. R. (1999). Dietary intake and biochemical, hematologic, and immune status of vegans compared with nonvegetarians. The American journal of clinical nutrition, 70(3), 586s-593s.

9. Vanacore D, Messina G, Lama S, Bitti G, Ambrosio P, Tenore G, Messina A, Monda V, Zappavigna S, Boccellino M, Novellino E, Monda M, Stiuso P. Effect of restriction vegan 
diet's on muscle mass, oxidative status, and myocytes differentiation: A pilot study $\mathrm{J}$ Cell Physiol 2018 Dec;233(12):9345-9353.

10. Gilsing AM, Crowe FL, Lloyd-Wright Z, Sanders TA, Appleby PN, Allen NE, Key TJ. Serum concentrations of vitamin B12 and folate in British male omnivores, vegetarians and vegans: results from a cross-sectional analysis of the EPIC-Oxford cohort study.Eur $\mathbf{J}$ Clin Nutr. 2010 Sep;64(9):933-9.

11. Merchant RE, Phillips TW, Udani J. Nutritional Supplementation with Chlorella pyrenoidosa Lowers Serum Methylmalonic Acid in Vegans and Vegetarians with a Suspected Vitamin B 12 Deficiency . J Med Food. 2015 Dec;18(12):1357-62

12. Craig WJ. Health effects of vegan diets. Am J Clin Nutr. 2009;89(5):1627S-1633S.

13. Schüpbach R, Wegmüller R, Berguerand C, Bui M, Herter-Aeberli I. Micronutrient status and intake in omnivores, vegetarians and vegans in Switzerland Eur J Nutr. 2017 Feb;56(1):283-293.

14. https://dietetycy.org.pl/dieta-weganska-porady-dla-sportowcow-osob-cwiczacych/ (Dostęp: 2020.07.21)

15. Fuhrman J, Ferreri DM. Fueling the vegetarian (vegan) athlete. Curr Sports Med Rep. 2010;9(4):233-241

16. Brown DD. Nutritional Considerations for the Vegetarian and Vegan Dancer. J Dance Med Sci 2018 Mar 15;22(1):44-53.

17. Craddock J, Probst Y, Peoples GE. Vegetarian and Omnivorous Nutrition Comparing Physical Performance. Int J Sport Nutr Exerc Metab. 2015.

18. Lynch HM,Wharton CM, Johnston CS. Cardiorespiratory Fitness and Peak Torque Differences between Vegetarian and Omnivore Endurance Athletes: A Cross-Sectional Study. Nutrients. 2016 Nov; 8(11): 726. 